\title{
Corrigendum: Cancer cells that survive radiation therapy acquire HIF-1 activity and translocate toward tumour blood vessels
}

Hiroshi Harada, Masahiro Inoue, Satoshi Itasaka, Kiichi Hirota, Akiyo Morinibu, Kazumi Shinomiya, Lihua Zeng, Guangfei Ou, Yuxi Zhu, Michio Yoshimura, W Gillies Mckenna, Ruth J. Muschel \& Masahiro Hiraoka

Nature Communications 3:783; doi: 10.1038/ncomms1786 (2012); Published 17 Apr 2012; Updated 16 Oct 2013

In Fig. 2c, d of this article, images corresponding to a time point of $16 \mathrm{~h}$ were inadvertently used to represent immunohistochemical labelling 3 days after tamoxifen injection. Images from the correct time point are included in the revised figure below. A revised version of Fig. 3 also follows, in which blood vessels are indicated by asterisks that were mistakenly deleted from panel a.
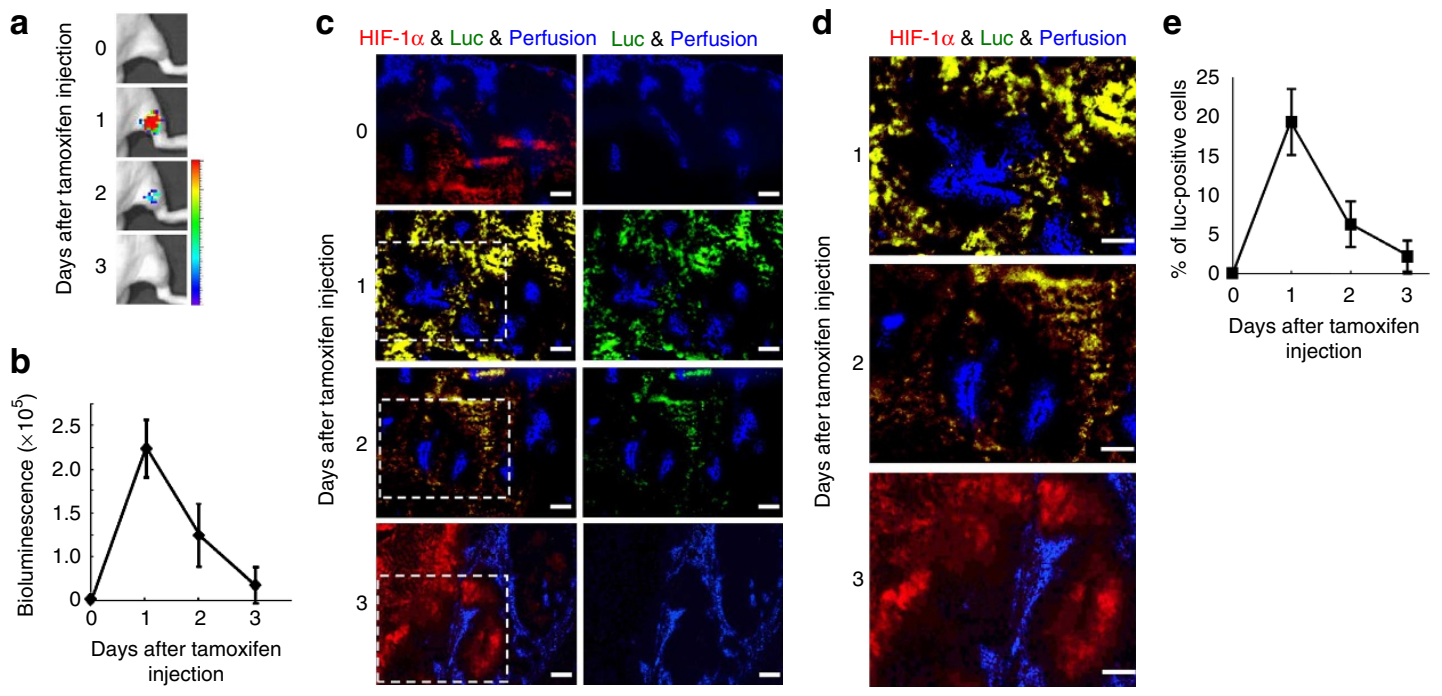

Figure 2 


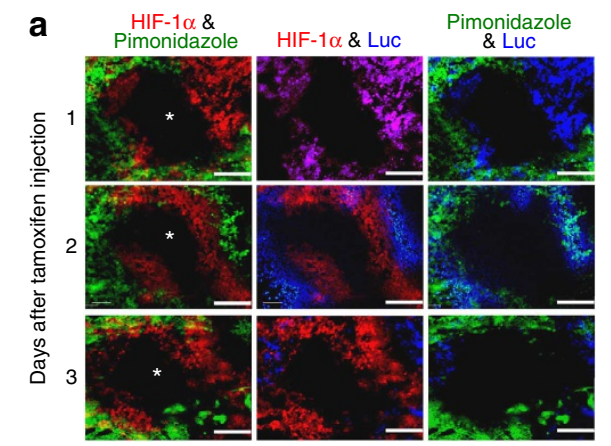

b

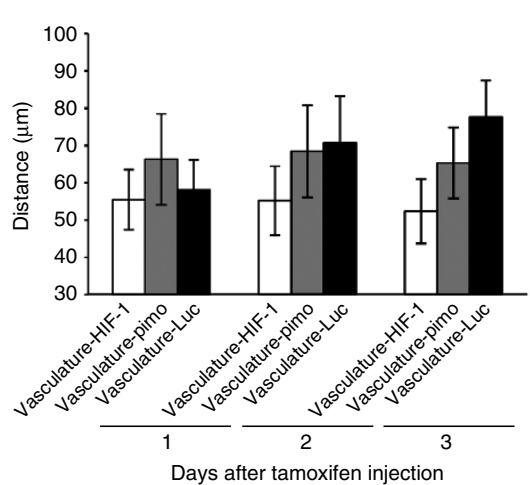

C

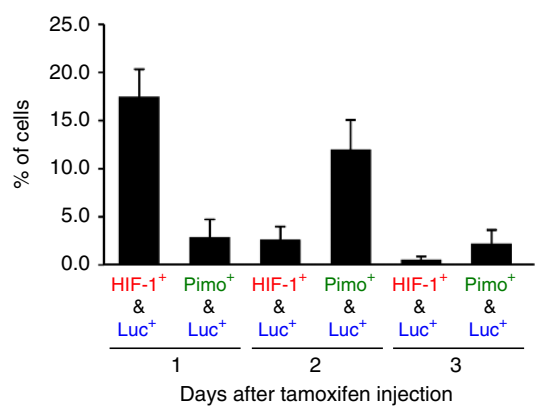

Figure 3 